\title{
ORIGINAL RESEARCH \\ Acute-Onset Migrainous Aura Mimicking Acute Stroke: MR Perfusion Imaging Features
}

D. Floery

M.R. Vosko

F.A. Fellner

C. Fellner

C. Ginthoer

F. Gruber

G. Ransmayr

A. Doerfler

M. Uder

W.G. Bradley

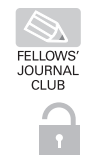

BACKGROUND AND PURPOSE: In a very limited number of cases, acute migrainous aura may mimic acute brain infarction. The aim of this study was to recognize patterns of MR perfusion abnormalities in this presentation.

MATERIALS AND METHODS: One thousand eight hundred fifty MR imaging studies performed for the suspicion of acute brain infarction were analyzed retrospectively to detect patients with acute migrainous aura not from stroke. All patients were examined clinically by 2 neurologists and underwent a standard stroke MR imaging protocol, including PWI. Two radiologists reviewed the perfusion maps visually and quantitatively for the presence, distribution, and grade of perfusion abnormalities.

RESULTS: Among $1850 \mathrm{MR}$ imaging studies, 20 (1.08\%) patients were found to have acute migrainous aura. Hypoperfusion was found in $14 / 20$ patients (70\%) with delayed rMTT and TTP, decreased rCBF, and minimal decrease in rCBV. In contrast to the typical pattern in stroke, perfusion abnormalities were not limited to a single vascular territory but extended to $>1$. Bilateral hypoperfusion was seen in $3 / 14$ cases. In 11/14 cases, hypoperfusion with a posterior predominance was found. TTP and rMTT were the best maps to depict perfusion changes at visual assessment, but also rCBF maps demonstrated significant hypoperfusion in quantitative analysis. In all patients, clinical and imaging follow-up findings were negative for stroke.

CONCLUSIONS: Acute migrainous aura is rare but important in the differential diagnosis among patients with the suspicion of acute brain infarction. Atypical stroke perfusion abnormalities can be seen in these patients.

ABBREVIATIONS: $\mathrm{ACA}=$ anterior cerebral artery; $\mathrm{PCA}=$ posterior cerebral artery; $\mathrm{rCBF}=$ relative cerebral blood flow; rCBV = relative cerebral blood volume; rMTT = relative mean transit time
D uring the past 10 years, MR imaging of acute stroke has become more and more important, culminating in the American Academy of Neurology updating their guidelines in July 2010, recommending DWI as the best method for the most accurate diagnosis.

Several other diseases, however, can mimic acute stroke, such as migrainous aura, which is a frequent neurologic disorder. Aura may manifest as acute neurologic symptoms before the onset of headache. ${ }^{1-5}$ Usual uncomplicated migraine attacks resolve after treatment. On the other hand, migraine with aura is a risk factor for ischemic stroke. ${ }^{6}$ The question is whether MR imaging is useful in the early distinction of migrainous aura and ischemic stroke.

Dedicated stroke MR imaging protocols have been established and include DWI and PWI as well as MRA of the intraand extracranial vessels. ${ }^{7-9}$ Whereas DWI is useful to depict the infarct core, PWI is used to determine the ischemic penumbra. ${ }^{10}$ The patterns of hypoperfusion in acute brain infarc-

Received October 27, 2011; accepted after revision November 17.

From the Institute of Radiology (D.F., F.A.F., C.G.) and Department of Neurology and Psychiatry (M.R.V., F.G., G.R.), AKH Linz, Linz, Austria; Institute of Radiology (C.F.), University Medical Center Regensburg, Regensburg, Germany; Department of Neuroradiology (F.A.F., A.D.) and Institute of Radiology (F.A.F., M.U.), University of Erlangen, Erlangen, Germany; and Department of Radiology (W.G.B.), University of California, San Diego, California.

Please address correspondence to Franz A. Fellner, MD, Institute of Radiology, AKH Linz, Krankenhausstr. 9, Linz 4020, Austria; e-mail: franz.fellner@akh.linz.at

Indicates open access to non-subscribers at www.ajnr.org

http://dx.doi.org/10.3174/ajnr.A3020 tion have been described. ${ }^{11}$ MR perfusion abnormalities have also been described in other disorders, (eg, seizures, vasospasm following subarachnoid hemorrhage, and severe stenosis of intra- or extracranial vessels). ${ }^{12,13}$

MR imaging, nuclear medicine, and CT perfusion studies have demonstrated that migrainous aura may be associated with perfusion abnormalities; however, all articles are limited to a small number of selected patients. ${ }^{14-24}$ To date, no data are available from a larger series of consecutive patients.

To our knowledge, this is the first study to describe perfusion anomalies-evaluated in a large cohort of 1850 consecutive acute stroke MR imaging examinations-in patients with acute migrainous aura mimicking acute stroke.

\section{Materials and Methods}

\section{Patients}

A retrospective analysis was performed during a 4-year period with a total of 1850 consecutive acute stroke MR imaging examinations (all including PWI). From this cohort, patients were selected in whom final neurologic and imaging work-up established the diagnosis of acute-onset migraine with aura.

Both the initial neurologic examination and the final diagnosis of migraine with aura or status migrainosus were performed by 2 neurologists according to established criteria. ${ }^{4}$ Patients in whom any other disease or condition was diagnosed that was considered as a possible complete or partial cause of the symptoms (history of previous brain infarction or transient ischemic attack or severe stenosis of intra- or extracranial vessels) were excluded. This was done to avoid any bias due to the inclusion of patients with other possible causes for 


\begin{tabular}{|c|c|c|c|c|c|c|c|}
\hline No. & Sequence & Orientation & $\begin{array}{c}\text { Section } \\
\text { Thickness } \\
(\mathrm{mm})\end{array}$ & $\begin{array}{c}\text { Pixel Size } \\
(\mathrm{mm} \times \mathrm{mm})\end{array}$ & $\begin{array}{c}\text { Contrast } \\
\text { Agent Flow } \\
\text { Rate }^{\mathrm{a}}\end{array}$ & $\begin{array}{c}\mathrm{TR} / \mathrm{TE} \\
\text { (ms) }\end{array}$ & $\begin{array}{l}\text { Acquisition } \\
\text { Time (min:s) }\end{array}$ \\
\hline 1 & DWI & Transverse & 6 & $1.1 \times 1.1$ & None & 4300/107 & 1:06 \\
\hline 2 & FLAIR & Transverse & 6 & $1.1 \times 0.9$ & None & 8390/121 & $1: 25$ \\
\hline 3 & 3D TOF & Transverse & 1 & $0.9 \times 0.9$ & None & 36/7.05 & 3:05 \\
\hline 4 & $\mathrm{~T} 2^{*} \mathrm{w}$ GRE & Transverse & 6 & $1.1 \times 0.9$ & None & $800 / 25.7$ & 1:14 \\
\hline 5 & PWI & Transverse & 6 & $2.0 \times 1.7$ & $\begin{array}{c}0.1 \mathrm{mmol} / \mathrm{kg} \\
5 \mathrm{~mL} / \mathrm{s}\end{array}$ & 2090/49 & 1:19 \\
\hline 6 & Neck MRA & Coronal & 1 & $1.4 \times 0.9$ & $\begin{array}{c}0.1 \mathrm{mmol} / \mathrm{kg} \\
2 \mathrm{~mL} / \mathrm{s}\end{array}$ & $3.27 / 1.17$ & $0: 49$ \\
\hline 7 & T1w postcontrast & Transverse & 6 & $1.2 \times 0.9$ & Postcontrast & $635 / 17$ & 1:37 \\
\hline
\end{tabular}

Note:-TOF indicates time-of-flight angiography (intracranial circulation);T2w, T2-weighted; T2* w GRE, T2*-weighted gradient-echo; T1w, T1-weighted

a Contrast dosage is given in millimole per kilogram of body weight.

abnormalities on PWI. Written informed consent was given by all patients. In a standardized review sheet, the clinical presentation was noted and the time interval between the onset of symptoms and the beginning of the MR imaging study was recorded. The study was approved by the institutional review board.

\section{Imaging Study}

All stroke MR imaging examinations were performed on 1.5T scanners (Magnetom Symphony or Magnetom Avanto; Siemens, Erlangen, Germany) with a maximum gradient strength between 30 and 45 $\mathrm{mT} / \mathrm{m}$ with a 12-channel standard head coil. Our MR imaging protocol using a free-induction-decay-EPI sequence for PWI is described in Table 1. The examinations including PWI were performed with full-head coverage ( 23 sections) in an axial orientation, with $\mathrm{TR}=$ $2090 \mathrm{~ms}, \mathrm{TE}=49 \mathrm{~ms}$, flip angle $=60^{\circ}, \mathrm{FOV}=220 \mathrm{~mm}$, matrix size $=$ $128 \times 96$ (read and phase), section thickness $=6 \mathrm{~mm}, 10 \%$ section gap, 1 average, parallel imaging factor $=2$. For PWI, 35 dynamic measurements (temporal resolution $=2090 \mathrm{~ms}$ for each dynamic series) were acquired after bolus injection (flow rate of $5 \mathrm{~mL} / \mathrm{s}$ ), followed by a $20-\mathrm{mL}$ saline flush of a $1.0-\mathrm{mol} / \mathrm{L}$ contrast agent, gadobutrol (Gadovist; Bayer HealthCare, Berlin, Germany). For extracranial angiography, a time-resolved contrast-enhanced MRA technique was used. Total measurement time was 10 minutes $25 \mathrm{sec}-$ onds for all sequences.

All PWI maps were calculated by using the same vendor-specific dedicated software (syngo Perfusion, version VB 17A; Siemens) by using a nonparametric singular value decomposition deconvolution technique. We calculated the following color-coded maps: TTP, rMTT, rCBV, and rCBF. For follow-up MR imaging (within 24 hours), the identical protocol was used.

\section{Image Analysis}

MR images were analyzed by 2 specialized neuroradiologists in consensus according to the established criteria. ${ }^{11}$ First, PWI was analyzed visually, with the readers blinded to all other sequences. After 1 week, the remaining sequences were analyzed and quantitative analysis of PWI maps was performed: In patients with a visually detected perfusion abnormality, a region of interest was defined in a representative section. The mean signal intensity was compared with an identical region of interest on the contralateral hemisphere (Fig 1). In patients with bilateral hypoperfusion, comparison was performed with normally perfused brain parenchyma in the MCA territory. In patients without visually detectable perfusion abnormalities, measurements were taken bilaterally in the MCA and PCA territories. For all patients, signal-intensity ratios (defined as the mean signal intensity in the affected brain region divided by mean signal intensity found in the normal hemisphere/region) were calculated. For visual assessment, perfusion abnormalities were classified as hyper- or hypoperfusion. Each abnormality was graded by using a 4-point scale: 1 , minor perfusion abnormality (subtle changes compared with the contralateral hemisphere); 2 , moderate perfusion abnormality; and 3, major perfusion abnormality. Grade 0 was the absence of a perfusion abnormality. Furthermore, the affected vascular territory was assessed.

\section{Statistical Analysis}

Statistical analysis was performed with the Statistical Package for the Social Sciences, Version 17 (SPSS, Chicago, Illinois). The Wilcoxon test was used to compare differences in the visual and quantitative assessment of perfusion maps; the Mann-Whitney $U$ test was applied to compare results in patients with and without perfusion abnormalities. A level of $P<.05$ was regarded as a statistically significant result. The Spearman rank correlation coefficient was calculated to compare results of the visual and quantitative assessments.

\section{Results}

Of 1850 stroke MR imaging examinations performed, 20 patients were found fulfilling the inclusion criteria ( 13 females, 7 males; mean age, 23 years; range, $17-51$ years). Thus, the incidence of migraine with aura was $20 / 1850(1.08 \%)$. None of the patients had a known history of previous attacks of migraine with focal neurologic deficits or other diseases.

Nineteen of 20 examinations were performed between 1 and 6 hours after onset (mean interval, 174 minutes). In 1 patient, the history of symptoms had persisted for 72 hours (status migrainosus) before the MR imaging examination. At the time of the examination, none of the patients had headache as the dominant symptom. There was a clear relationship between the perfusion abnormality and the symptoms of the patients. In the patients with posterior predominance of the hypoperfusion, visual deterioration and nausea or vertigo or both were seen. For example, of 11 patients with visual symptoms, 9 patients had hypoperfusion in the area of the PCA. Furthermore, the patients with motor deficits or paresthesias showed perfusion abnormalities in the area of the MCA. Eight of 9 patients with aphasia demonstrated hypoperfusion in the area of the MCA (Table 2).

In 14 patients (70.0\%), hypoperfusion was found by visual assessment, unilateral in $11 / 14(78.6 \%)$ and bilateral in $3 / 14$ $(21.4 \%)$ patients. In $10 / 14(71.4 \%)$ patients, a posterior predominance of hypoperfusion was seen-located in the PCA 

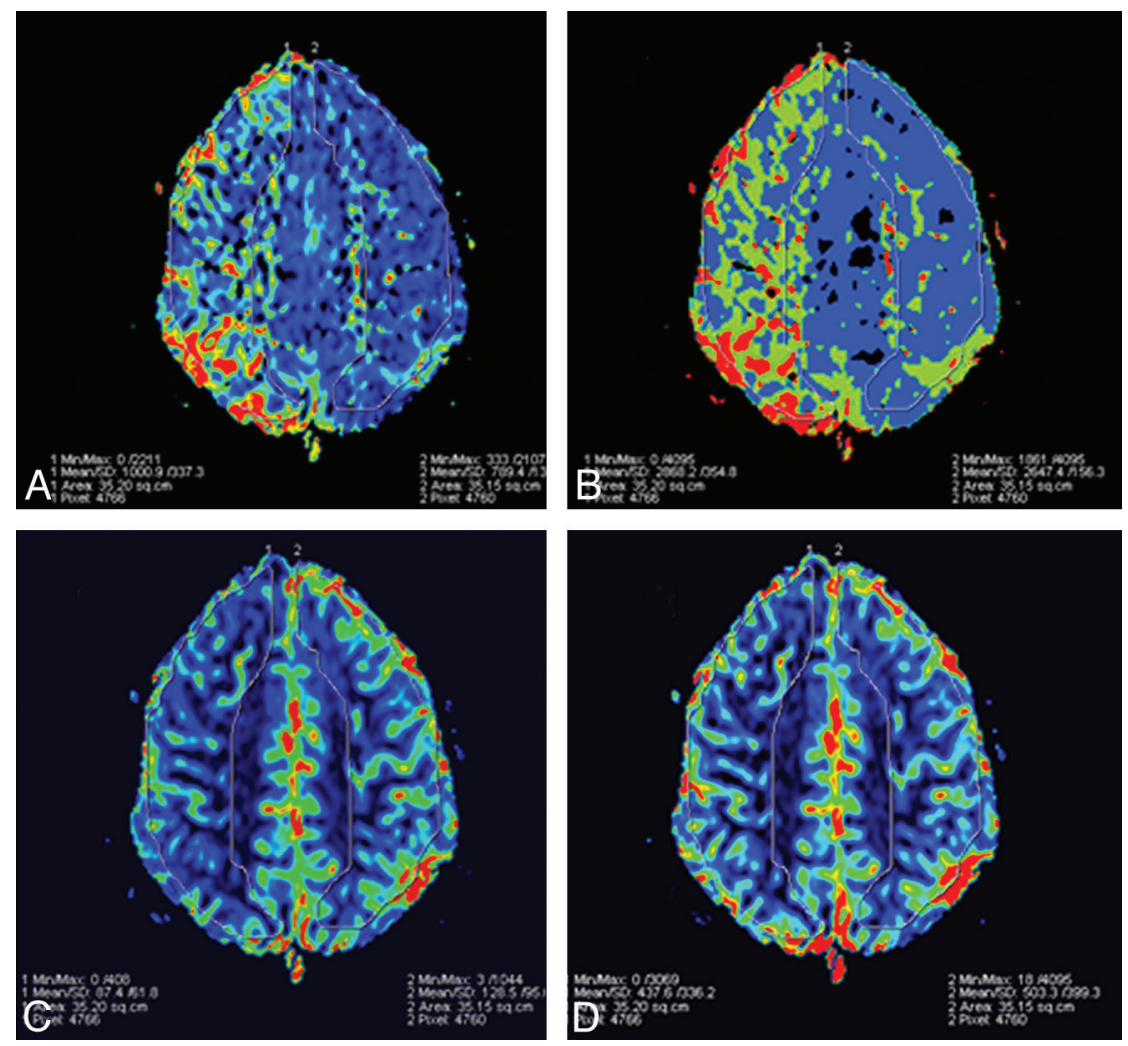

Fig 1. Quantitative evaluation of perfusion maps with region-of-interest measurements of the affected and normal side. $A, r M T T$. $B$, TTP. $C$, rCBF, $D$, rCBV

\begin{tabular}{|c|c|c|c|c|c|c|c|c|c|c|c|c|c|c|c|c|c|c|c|c|}
\hline \multirow[b]{2}{*}{ No. } & \multirow{2}{*}{$\begin{array}{c}\text { Perfusion } \\
\text { Abnormality }\end{array}$} & \multirow{2}{*}{$\begin{array}{l}\text { Time to } \\
\text { Imaging }^{\mathrm{a}}\end{array}$} & \multicolumn{6}{|c|}{ Clinical Symptoms ${ }^{\mathrm{b}}$} & \multicolumn{3}{|c|}{$\mathrm{TTP}^{\mathrm{c}}$} & \multicolumn{3}{|c|}{ rMTT } & \multicolumn{3}{|c|}{ rCBF } & \multicolumn{3}{|c|}{ rCBV } \\
\hline & & & VS & $\mathrm{AP}$ & PA & $\mathrm{MD}$ & $\mathrm{N}$ & $\mathrm{V}$ & Ant & Med & Post & Ant & Med & Post & $\overline{\text { Ant }}$ & Med & $\overline{\text { Post }}$ & Ant & Med & Post \\
\hline 1 & Yes & 60 & 1 & 1 & 0 & 0 & 1 & 0 & 0 & 3 & 3 & 0 & 2 & 2 & 0 & 2 & 2 & 0 & 1 & 1 \\
\hline 2 & Yes & 60 & 0 & 1 & 1 & 1 & 0 & 0 & 0 & 3 & 3 & 0 & 2 & 2 & 0 & 2 & 2 & 0 & 1 & 1 \\
\hline 3 & No & 360 & 0 & 0 & 1 & 0 & 1 & 0 & 0 & 0 & 0 & 0 & 0 & 0 & 0 & 0 & 0 & 0 & 0 & 0 \\
\hline 4 & No & 240 & 1 & 1 & 0 & 0 & 0 & 1 & 0 & 0 & 0 & 0 & 0 & 0 & 0 & 0 & 0 & 0 & 0 & 0 \\
\hline 5 & Yes & 60 & 1 & 0 & 1 & 0 & 0 & 0 & 0 & 3 & 3 & 0 & 2 & 2 & 0 & 2 & 2 & 0 & 1 & 1 \\
\hline 6 & Yes & 240 & 1 & 0 & 0 & 0 & 0 & 0 & 0 & 3 & 3 & 0 & 3 & 3 & 0 & 0 & 0 & 0 & 0 & 0 \\
\hline 7 & No & 300 & 0 & 0 & 1 & 0 & 1 & 0 & 0 & 0 & 0 & 0 & 0 & 0 & 0 & 0 & 0 & 0 & 0 & 0 \\
\hline 8 & Yes & 210 & 1 & 1 & 1 & 0 & 0 & 0 & 3 & 3 & 3 & 2 & 2 & 2 & 2 & 2 & 2 & 1 & 1 & 1 \\
\hline 9 & Yes & 198 & 1 & 1 & 1 & 0 & 1 & 1 & 0 & 3 & 3 & 0 & 3 & 3 & 0 & 2 & 2 & 0 & 1 & 1 \\
\hline 10 & No & 120 & 1 & 0 & 0 & 0 & 0 & 0 & 0 & 0 & 0 & 0 & 0 & 0 & 0 & 0 & 0 & 0 & 0 & 0 \\
\hline 11 & Yes & 100 & 0 & 1 & 1 & 0 & 0 & 0 & 0 & 2 & 2 & 0 & 1 & 1 & 0 & 1 & 1 & 0 & 1 & 1 \\
\hline 12 & Yes & 100 & 1 & 0 & 1 & 1 & 1 & 1 & 3 & 3 & 3 & 3 & 3 & 3 & 2 & 2 & 2 & 1 & 1 & 1 \\
\hline 13 & Yes & 60 & 0 & 1 & 0 & 0 & 0 & 0 & 3 & 3 & 3 & 3 & 3 & 3 & 2 & 2 & 2 & 1 & 1 & 1 \\
\hline 14 & Yes & $<6 \mathrm{hr}$ & 0 & 1 & 0 & 0 & 1 & 0 & 3 & 3 & 3 & 2 & 2 & 2 & 1 & 1 & 1 & 0 & 0 & 0 \\
\hline 15 & Yes & 80 & 1 & 0 & 0 & 0 & 1 & 1 & 0 & 1 & 1 & 0 & 0 & 0 & 0 & 0 & 0 & 0 & 0 & 0 \\
\hline 16 & Yes & $72 \mathrm{hr}$ & 1 & 0 & 0 & 0 & 1 & 1 & 0 & 3 & 3 & 0 & 1 & 1 & 0 & 1 & 1 & 0 & 0 & 0 \\
\hline 17 & Yes & $<6 \mathrm{hr}$ & 1 & 0 & 0 & 0 & 1 & 1 & 0 & 3 & 3 & 0 & 1 & 1 & 0 & 1 & 1 & 0 & 1 & 1 \\
\hline 18 & No & 360 & 0 & 0 & 1 & 1 & 0 & 0 & 0 & 0 & 0 & 0 & 0 & 0 & 0 & 0 & 0 & 0 & 0 & 0 \\
\hline 19 & No & 210 & 0 & 0 & 1 & 0 & 0 & 0 & 0 & 0 & 0 & 0 & 0 & 0 & 0 & 0 & 0 & 0 & 0 & 0 \\
\hline 20 & Yes & 200 & 0 & 1 & 0 & 0 & 0 & 0 & 0 & 3 & 3 & 0 & 2 & 2 & 0 & 2 & 2 & 0 & 1 & 1 \\
\hline \multicolumn{9}{|c|}{ Mean grade score } & 0.60 & 1.95 & 1.95 & 0.50 & 1.40 & 1.40 & 0.35 & 1.00 & 1.00 & 0.15 & 0.50 & 0.50 \\
\hline \multicolumn{9}{|c|}{ Total mean grade score } & & 1.50 & & & 1.10 & & & 0.78 & & & 0.38 & \\
\hline
\end{tabular}

Note:-VS indicates visual symptoms; AP, aphasia; PA, paresthesias; MD, motor disabilities; N: nausea; V, vertigo; Ant, anterior; Med, middle; Post, posterior.

a Time to imaging is the interval between onset of symptoms and beginning of MRI study, given in minutes.

${ }^{b}$ History of migraine is the available clinical history of migraine at the time of initial neurologic examination.

${ }^{c}$ Perfusion abnormalities on visual assessment for all patients according to the vascular territories using a 4-point scale $(0=$ no abnormality, $3=$ major abnormality).

territory and in varying parts of the MCA territory. In the remaining 4 patients $(28.5 \%)$, hypoperfusion involved all 3 vascular territories. Graded visual assessment of hypoperfusion is shown in Table 2.
At visual assessment, hypoperfusion was significantly more pronounced on TTP than on $\operatorname{rMTT}(P=.004), \operatorname{rCBV}(P<$ $.001)$, or $\operatorname{rCBF}(P<.001)$ maps. rMTT was more sensitive than $\operatorname{rCBV}(P=.001)$ and $\operatorname{rCBF}(P=.031)$. Notably, rCBF 
Table 3: Mean signal ratios and SD for quantitative and visual assessment of PWI: separated for cases with and without visually detected perfusion abnormalities ${ }^{a}$

\begin{tabular}{lccc}
\hline & Cases with Visually Detected Perfusion Abnormalities & $\begin{array}{c}\text { Cases without Visually Detected } \\
\text { Perfusion Abnormalities }\end{array}$ \\
\cline { 2 - 4 } & $\begin{array}{c}\text { Visual Score, grade 0-3 } \\
\text { (mean } \pm \text { SD) }\end{array}$ & $\begin{array}{c}\text { Signal Ratio } \\
\text { (mean } \pm \text { SD) }\end{array}$ & $\begin{array}{c}\text { Signal Ratio } \\
\text { (mean } \pm \text { SD) }\end{array}$ \\
\hline TTP & $2.14 \pm 0.68$ & $1.08 \pm 0.02$ & $0.99 \pm 0.01$ \\
IMTT & $1.57 \pm 0.88$ & $1.31 \pm 0.19$ & $1.02 \pm 0.04$ \\
rCBF & $1.12 \pm 0.66$ & $0.64 \pm 0.16$ & $1.03 \pm 0.07$ \\
rCBV & $0.55 \pm 0.38$ & $0.83 \pm 0.18$ & $1.05 \pm 0.07$ \\
\hline
\end{tabular}

a Signal ratio of the quantitative assessment is calculated as the signal of the affected side divided by the signal of the normal side; in cases without visually detected perfusion abnormality, it means the signal of the left divided by the signal of the right side.
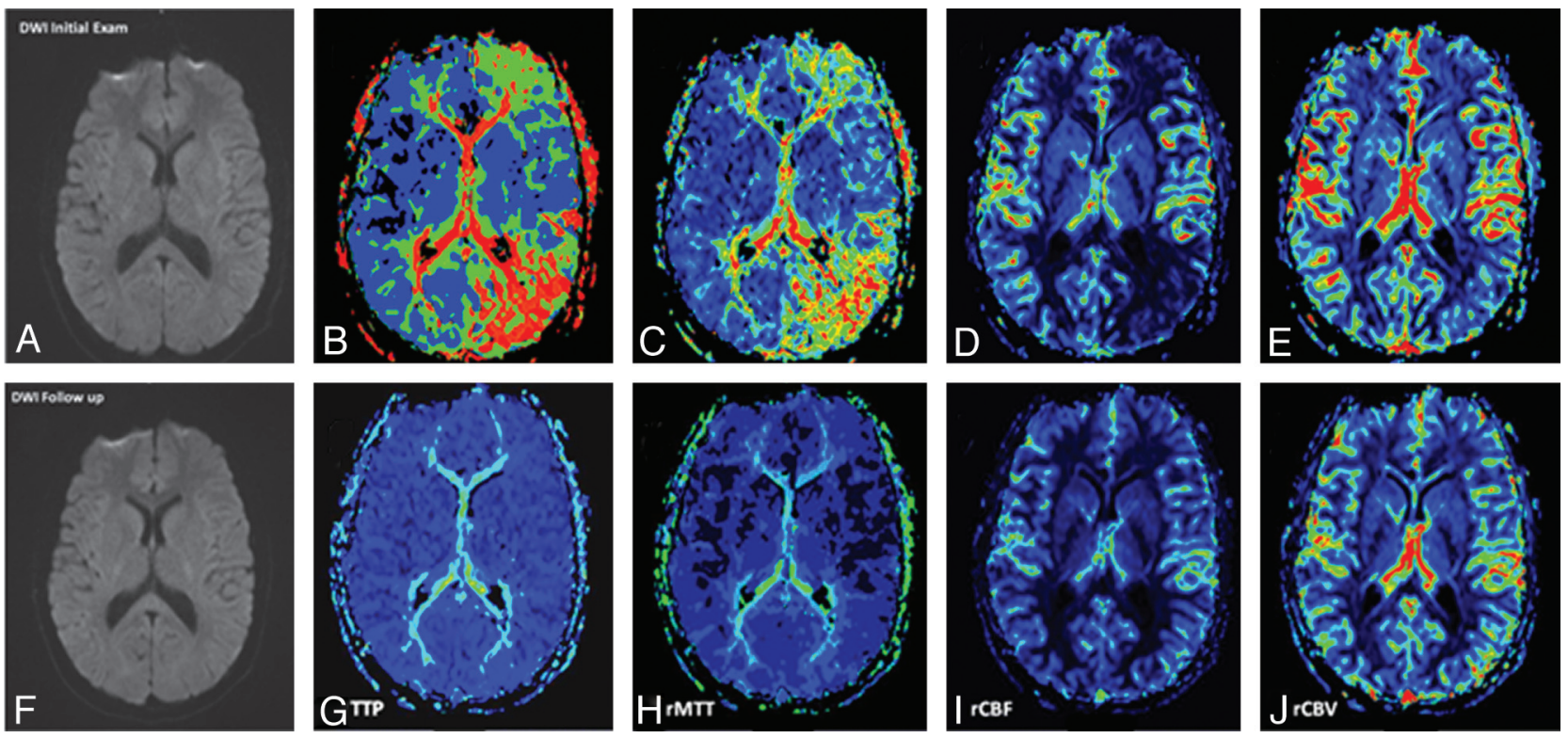

Fig 2. A-F, A 17-year-old male patient with acute onset of aphasia and right-sided paresthesias: DWI findings were negative for ischemic brain infarction initially $(A)$ and during follow-up (F). B-E, PWI demonstrates hypoperfusion in the posterior cerebral and, to a lesser degree, in the middle cerebral vascular territories on the left side-most pronounced in TTP (grade 3) and rMTT (grade 2) maps. rCBF maps demonstrated hypoperfusion grade 2; rCBV maps demonstrated hypoperfusion grade 1. G-J, Follow-up PWI after 24 hours shows complete resolution of hypoperfusion.

also showed hypoperfusion in 13/14 cases, though it was significantly less pronounced than rMTT and TTP. rCBV only showed subtle hypoperfusion in 10/14 cases.

At quantitative analysis, the following mean signal-intensity ratios were found in patients demonstrating hypoperfusion at visual assessment: TTP, 1.08; rMTT, 1.31; rCBF, 0.64; $\mathrm{rCBV}, 0.83$. Mean signal intensity ratios for patients without visually detected perfusion abnormalities are given in Table 3. Quantitatively, the most pronounced changes were found in rCBF followed by $\operatorname{rMTT}(P<.001), \operatorname{rCBV}(P<.001)$, and TTP $(P<.001)$. Differences between patients with and without visually observed hypoperfusion were significant for all maps.

When we compared visual and quantitative assessments, the Spearman correlation coefficient revealed significant correlation for TTP $(r=0.81)$, $\operatorname{rMTT}(r=0.84)$, and $\operatorname{rCBF}(r=$ $-0.72)$; for $\mathrm{rCBV}$, the correlation was not significant $(r=$ $-0.46)$.

No other sequences showed any pathologic findings. In particular, there was no evidence of restricted diffusion (Fig 2).

Clinical follow-up was available in all cases. In all patients, symptoms fully resolved, spontaneously $(n=5)$ or after treat- ment with IV acetylsalicylic acid or acetaminophen $(n=14)$. Systemic thrombolysis (alteplase) was performed in 1 patient with suspected stroke at the beginning of the study period (the first patient with migraine in this study). In 14/20 patients, follow-up examinations in a time interval of 20-30 hours (mean, 22 hours) were available. All follow-ups demonstrated complete resolution of hypoperfusion at visual assessment (Figs 2-4). Repeat standard sequences demonstrated no change to the initial examination.

\section{Discussion}

A subset of migraine attacks may manifest with acute neurologic symptoms (ie, aura) that can also be found in acute stroke. ${ }^{1}$ These symptoms occur before the migrainous headache. Cases with predominantly motor symptoms are also referred to as "hemiplegic migraine." ${ }^{25}$ According to the International Headache Society, ${ }^{4}$ these forms are diagnosed clinically by accurate patient history and neurologic examination. However, substantial problems may arise in the acute phase because accurate information is often incomplete or difficult to obtain. ${ }^{3}$ Distinguishing between migrainous aura, cerebral ischemia, and Todd paralysis following a seizure may be difficult. In our series, no one had a previous history of 

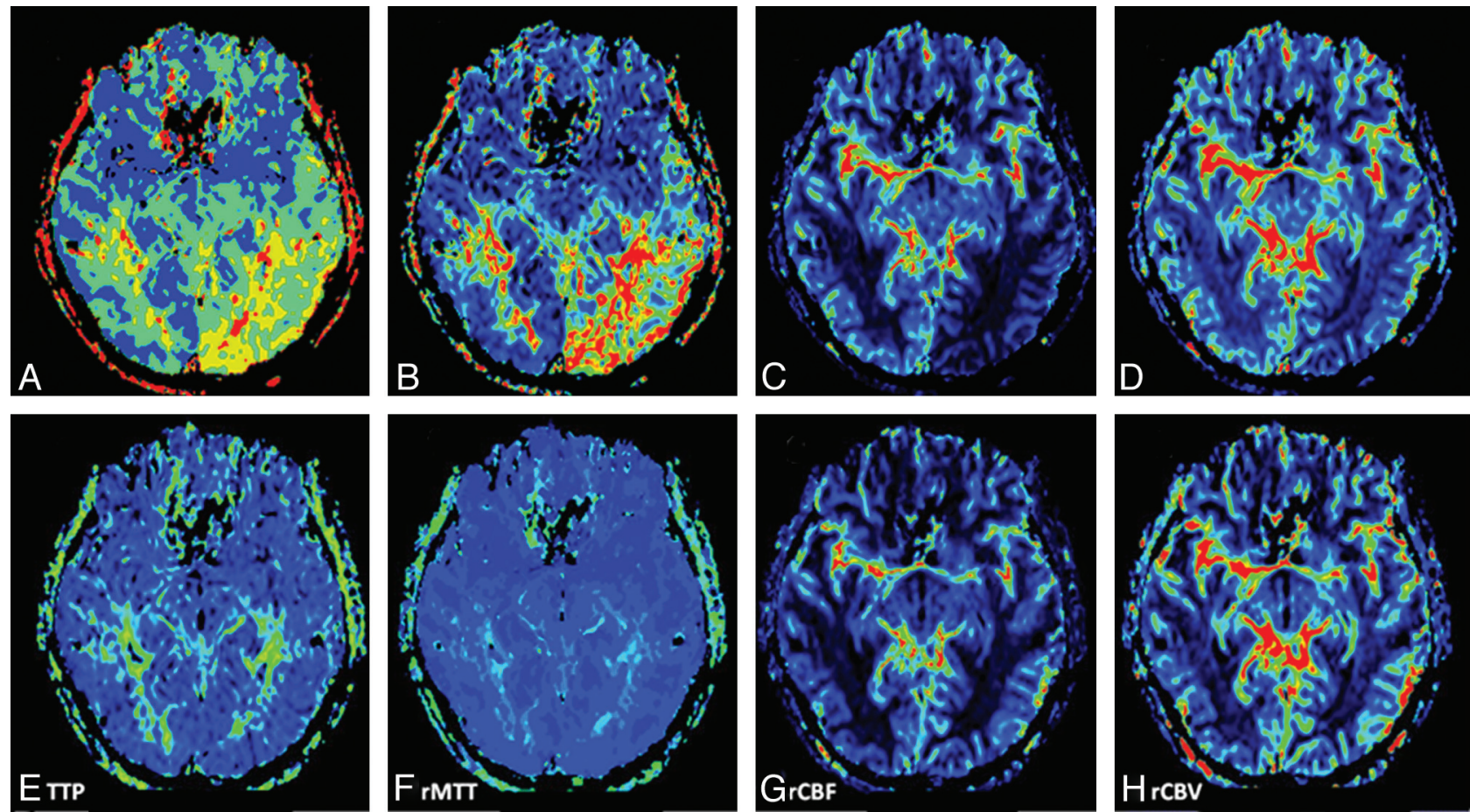

Fig 3. A 24-year-old male patient presenting with acute-onset aphasia, visual reduction, and right-sided paresthesias. Initial PWI ( $A-D)$ shows apparent hypoperfusion, best outlined in rMTT (grade 3) and TTP (grade 3). rCBF maps demonstrate hypoperfusion grade 2. In rCBV maps, no visible hypoperfusion is demonstrated. E-H, Follow-up PWI after 24 hours shows complete resolution of hypoperfusion in all maps.
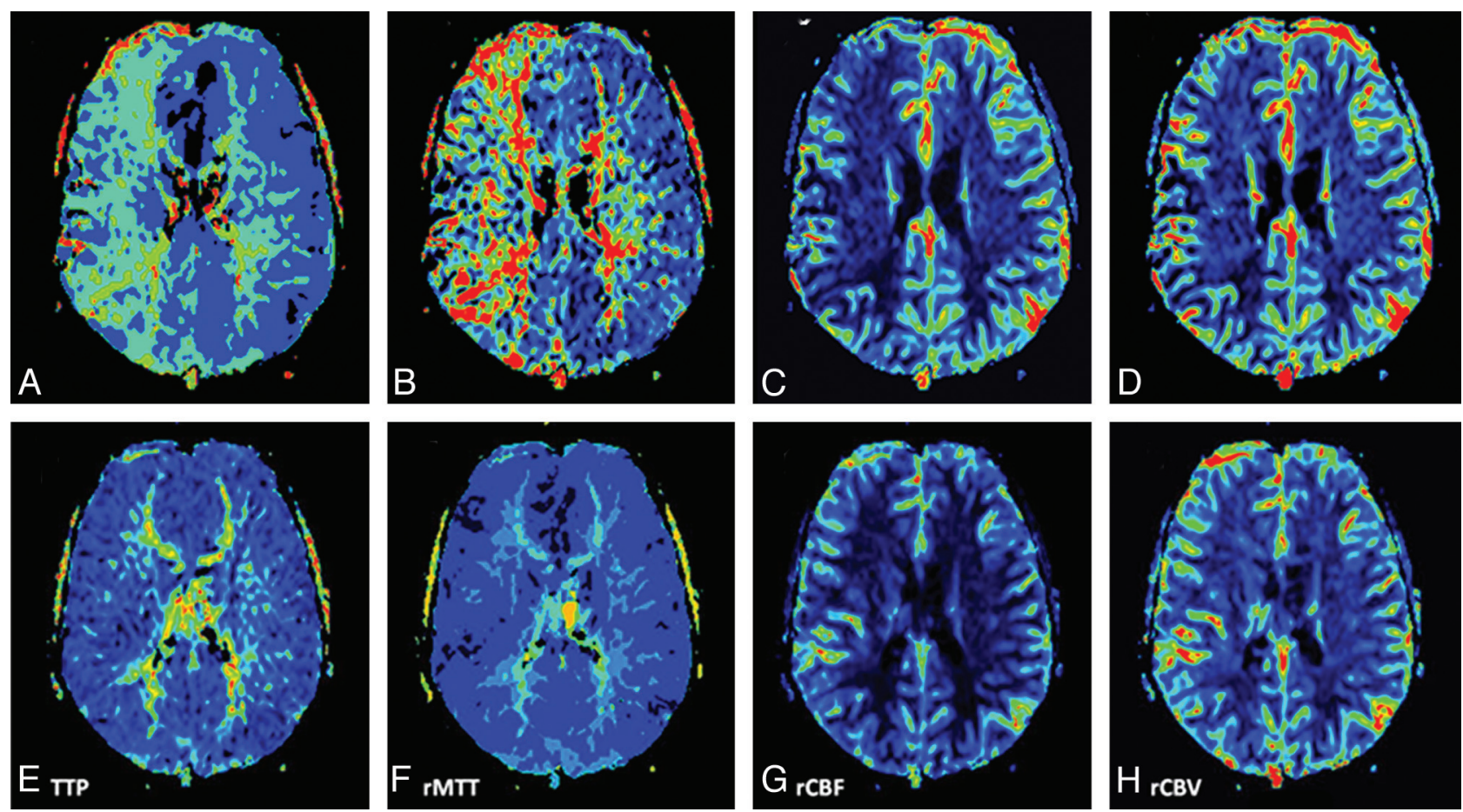

Fig 4. A 19-year-old female patient presenting with scintillating scotomata and left-sided hemiparesis and hemisensory symptoms. $A-D$, PWI 100 minutes after the onset of symptoms demonstrates hypoperfusion in the entire right hemisphere, delineated on rMTT (grade 3) and TTP (grade 3), rCBF (grade 2), and rCBV (grade 1). Follow-up PWI after 28 hours and after treatment with IV salicylates ( $E-H)$ shows no remaining hypoperfusion.

migraine with acute neurologic deficits. Thus, a certain dilemma arises because an accurate differential diagnosis is mandatory for correct treatment.

Stroke MR imaging has demonstrated its clinical importance in the work-up of acute brain infarction. ${ }^{7,26}$ Perfusion abnormalities in acute brain infarction usually comprise hypoperfusion in a defined (mostly single) vascular territory. ${ }^{11}$

There are reports that certain perfusion abnormalities may be found in migraine by using PWI; however, these are limited to case studies. ${ }^{17-19}$ 
Since 2005, we have examined all patients with acute stroke who are potentially candidates for thrombolysis or mechanical intervention, primarily with stroke MR imaging. From 1850 consecutive patients with acute stroke symptoms, 20 patients with migraine that mimicked acute stroke $(1.08 \%$ of all patients) were found. In the case of standard unenhanced CT, these patients would probably have been treated with systemic thrombolysis, which is risky and delays specific antimigrainous treatment.

Perfusion abnormalities were found in 70\% (14/20 patients). That implies that - if migrainous aura is present-perfusion abnormalities are frequent. In each case, the perfusion abnormality was detected by visual evaluation, which was performed in the first step of the study evaluation. Subsequent quantitative analysis did not reveal any further cases with significant perfusion abnormalities. This finding demonstrates that visual assessment, which is usually performed in the acute setting, also seems to be adequate to show migraine-associated perfusion abnormalities. Quantitative assessment-which takes additional time-does not seem to be mandatory in the acute setting.

Of these 14 cases showing perfusion abnormalities, increased perfusion was not seen in any. In our opinion, this was due to the fact that all patients were examined in the stage of migrainous aura, where neurologic deficits-but not headache-were the dominant symptom. Even in 1 patient with a prolonged symptom history for 3 days, no headache was present, similar to findings in other reports. ${ }^{16}$ Some published studies report hyperperfusion; however, this is during the headache stage, 6-24 hours after the onset of symptoms. ${ }^{2,17,19-21,24}$ Others report hypoperfusion during the migrainous aura. ${ }^{2,14,18,22,23,27}$

These findings emphasize that early MR imaging examination is crucial to detect the perfusion abnormalities. This correlates with the presumed pathogenesis of migraine, which includes a combination of vascular and neuronal factors. ${ }^{28,29}$ The finding of occipital predominance of vasoconstriction during the aura is supported by our data because we found a posterior predominance of hypoperfusion $(71.4 \%)$.

The striking point is that in all cases, $>1$ vascular territory was affected, which holds the greatest potential to differentiate migraine from stroke, where hypoperfusion is usually limited to a single vascular territory. In 4 of 14 patients $(28.6 \%)$, the entire hemisphere was hypoperfused. A possible differential diagnosis for this pattern of hypoperfusion is severe stenosis of the extracranial vessels. However, this can be ruled out by MRA of the extracranial vessels.

Concerning the 4 PWI maps, we found that quantitative evaluation of the perfusion abnormalities was not much more striking than visual inspection. In the visual assessment, TTP was significantly superior, followed by rMTT (Table 2). TTP and rMTT were positive for hypoperfusion in all positive cases except 1 , in which only subtle findings (grade 1 ) were seen in TTP, with the other maps having negative findings. Notably, all except 1 of the cases positive for hypoperfusion also showed changes in $\mathrm{rCBF}^{21,27}$ On quantitative analysis, $\mathrm{rCBF}$ demonstrated the most pronounced changes, followed by rMTT and TTP. The Spearman correlation test revealed significant correlation for TTP, rMTT, and rCBF between visual and quantitative analysis, except for rCBV. This may be due to the col- or-coding of the lookup table in combination with window and center settings that we use that encode the rCBF map predominantly with dark colors, blue and black. However, the quantitative results can be better appreciated if the rCBF images are windowed and centered with extremely narrow values. Of course, in the acute clinical setting, visual evaluation is used first because it is the fastest approach ("time is brain"). In cases of doubt, however, additional quantitative evaluation with calculation of ratios in the different maps between the affected and the normal hemisphere may be performed.

In all patients, clinical follow-up was available. All showed complete resolution of the symptoms, spontaneously or after treatment with analgesics. MR imaging follow-up was performed in 14/20 patients (70\%) and showed complete resolution of hypoperfusion in all cases.

A limitation of this study is its retrospective design, describing only patterns of perfusion anomalies. Sensitivities or specificities cannot be provided and must be the topic of further research. PWI in patients with migraine during headache will be a topic of further research in a prospective setting. The comparison of visual-versus-quantitative assessment of PWI also warrants further research.

\section{Conclusions}

The American Academy of Neurology updated their guidelines in July 2010, recommending DWI as the superior method (level A) for the most accurate stroke diagnosis. ${ }^{30}$ The study presented here supports that recommendation and demonstrates the potential of PWI in eliminating differential considerations.

To our knowledge, this is the first study to describe perfusion anomalies-evaluated with acute stroke MR imaging - in patients with acute migrainous aura mimicking acute stroke. Migraine is a rare but important differential diagnosis among patients presenting with symptoms of acute stroke. Hypoperfusion affecting $>1$ vascular territory is the most important finding on PWI, which can lead one to the correct diagnosis and therapy, particularly if no diffusion restriction is seen.

Disclosures: Michael Uder-UNRELATED: Payment for Lectures (including service on speakers bureaus): Bracco, Siemens, Medtronic.

\section{References}

1. Foroozan R, Cutrer FM. Transient neurologic dysfunction in migraine. Neurol Clin 2009;27:361-78

2. Baron JC. Stroke: imaging and differential diagnosis. J Neural Transm Suppl 2002;63:19-36

3. Schoenen J, Sandor PS. Headache with focal neurological signs or symptoms: a complicated differential diagnosis. Lancet Neurol 2004;3:237-45

4. Solomon S. Diagnosis of primary headache disorders: validity of the International Headache Society criteria in clinical practice. Neurol Clin 1997;15:15-26

5. Wardlaw JM, Murray V, Berge E, et al. Thrombolysis for acute ischaemic stroke. Cochrane Database Syst Rev 2009:CD000213

6. Pezzini A, Del Zotto E, Giossi A, et al. The migraine-ischemic stroke connection: potential pathogenic mechanisms. Curr Mol Med 2009;9:215-26

7. Harris AD, Coutts SB, Frayne R. Diffusion and perfusion MR imaging of acute ischemic stroke. Magn Reson Imaging Clin N Am 2009;17:291-313

8. Olivot JM, Marks MP. Magnetic resonance imaging in the evaluation of acute stroke. Top Magn Reson Imaging 2008;19:225-30

9. Donnan GA, Davis SM. Neuroimaging, the ischaemic penumbra, and selection of patients for acute stroke therapy. Lancet Neurol 2002;1:417-25

10. Ostergaard L. Principles of cerebral perfusion imaging by bolus tracking. $J$ Magn Reson Imaging 2005;22:710-17 
11. Srinivasan A, Goyal M, Al Azri F, et al. State-of-the-art imaging of acute stroke. Radiographics 2006;26(suppl 1):S75-95

12. Forsting M, Weber J. MR perfusion imaging: a tool for more than stroke. Eur Radiol 2004;14(suppl 5):M2-7

13. Huang BY, Castillo M. Radiological reasoning: extracranial causes of unilateral decreased brain perfusion. AJR Am J Roentgenol 2007;189(6 suppl):S49-54

14. Cheng MF, Wu YW, Tang SC. Cerebral perfusion changes in hemiplegic migraine: illustrated by Tc-99m ECD brain perfusion scan. Clin Nucl Med 2010;35:456-58

15. Cutrer FM, Sorensen AG, Weisskoff RM, et al. Perfusion-weighted imaging defects during spontaneous migrainous aura. Ann Neurol 1998;43:25-31

16. Jager HR, Giffin NJ, Goadsby PJ. Diffusion- and perfusion-weighted MR imaging in persistent migrainous visual disturbances. Cephalalgia 2005;25: 323-32

17. Lindahl AJ, Allder S, Jefferson D, et al. Prolonged hemiplegic migraine associated with unilateral hyperperfusion on perfusion weighted magnetic resonance imaging. J Neurol Neurosurg Psychiatry 2002;73:202-03

18. Linn J, Freilinger T, Morhard D, et al. Aphasic migrainous aura with left parietal hypoperfusion: a case report. Cephalalgia 2007;27:850-53

19. Masuzaki M, Utsunomiya $H$, Yasumoto $S$, et al. A case of hemiplegic migraine in childhood: transient unilateral hyperperfusion revealed by perfusion MR imaging and MR angiography. AJNR Am J Neuroradiol 2001;22:1795-97

20. Oberndorfer S, Woeber C, Nasel C, et al. Familial hemiplegic migraine: follow-up findings of diffusion-weighted magnetic resonance imaging (MRI), perfusion-MRI and [99mTc] HMPAO-SPECT in a patient with prolonged hemiplegic aura. Cephalalgia 2004;24:533-39

21. Pollock JM, Deibler AR, Burdette JH, et al. Migraine associated cerebral hyper- perfusion with arterial spin-labeled MR imaging. AJNR Am J Neuroradiol 2008;29:1494-97

22. Relja G, Granato A, Ukmar M, et al. Persistent aura without infarction: description of the first case studied with both brain SPECT and perfusion MRI. Cephalalgia 2005;25:56-59

23. Yamada K, Harada M, Inoue N, et al. Concurrent hemichorea and migrainous aura: a perfusion study on the basal ganglia using xenon-computed tomography. Mov Disord 2008;23:425-29

24. Yamashita S, Hamano S, Tanaka M, et al. Single photon emission computed tomography findings in a case of alternating hemiplegia of childhood in relation to migraine [in Japanese]. No To Hattatsu 2005;37:413-18

25. Black DF. Sporadic hemiplegic migraine. Curr Pain Headache Rep 2004;8: 223-28

26. Tomandl BF, Klotz E, Handschu R, et al. Comprehensive imaging of ischemic stroke with multisection CT. Radiographics 2003;23:565-92

27. Kato $Y$, Araki N, Matsuda H, et al. Arterial spin-labeled MRI study of migraine attacks treated with rizatriptan. J Headache Pain 2010;11:255-58. Epub 2010 Apr 22

28. Olesen J, Friberg L, Olsen TS, et al. Timing and topography of cerebral blood flow, aura, and headache during migraine attacks. Ann Neurol 1990;28:791-98

29. Silberstein SD. Migraine pathophysiology and its clinical implications. Cephalalgia 2004;24(suppl 2):2-7

30. Schellinger PD, Bryan RN, Caplan LR, et al. Evidence-based guideline: the role of diffusion and perfusion MRI for the diagnosis of acute ischemic strokereport of the Therapeutics and Technology Assessment Subcommittee of the American Academy of Neurology. Neurology 2010;75:177-85 